\title{
SLEEP AND THE ATHLETE
}

\author{
C. M. SHAPIRO, MB, BCh, BSc(Hon)
}

Department of Psychiatry, Royal Edinburgh Hospital, Edinburgh \begin{abstract}
totally supportive of the restorative theory of sleep function. manifestations of this sleep deprivation. aspects of athletic performance.

Sleep is the period that is popularly recognised as a time when the body is "restored". This popular recognition has only in the past ten years gained scientific credibility. One of the approaches that have been used to look at the question of sleep function is to study the effect of exercise on sleep. These studies have produced superficially contradictory results, but I hope to show that the majority of these studies show an effect of exercise on sleep consistent with the theory that sleep is a recovery process for daytime activity.
\end{abstract}

ABSTRACT

Sleep is generally considered to be restorative and the notion of exercise being associated with the changes in subsequent sleep is popular but has only recently been demonstrated. There are several facets of exercise performed that have an influence on sleep. These include the intensity and duration of the exercise, and the interval between the cessation of exercise and sleep onset. Other factors that may alter sleep after exercise are the age and fitness of the subject, and his lean body mass. Most studies on the effect of exercise on sleep can be interpreted as being partially or

Sleep deprivation alters performance and one may expect that, as with other physiological parameters that have a normal range, certain sleep patterns may be conducive to better athletic performance. Sleep patterns may be influenced by physical training. Extreme endurance events result in sleep deprivation. It is important to identify the clinical

The interaction of exercise and sleep has implications for both theoretical models of sleep function and practical

Unfortunately there are few studies on the effect of sleep (or sleep loss) on athletic performance. There are many studies on the effect of sleep (and sleep lack) on performance and vigilance and some of these are summarised briefly, as it is reasonable to expect that the conclusions that can be drawn from them can be extrapolated to athletic performance.

There are almost 30 studies in the literature on the effect of exercise on sleep. From these studies it is difficult to establish definite changes that occur during sleep after exercise, as these experiments have had several variables, which differed in the different experiments. These differences have included: the intensity and duration of the exercise; the interval between cessation of exercise and sleep onset; fitness of subjects; lean body mass; and subjects' age ranges. There are reasons to be found in the literature to suggest that all these variables may have some bearing on the effect that exercise has on sleep. It would seem that the most important variable is that of intensity of exercise. There are many reports in which work load is not described, but most reports give some perspective of the total amount of exercise performed. The range is wide and includes isometric exercise (" 80 minutes at $40 \%$ of maximal level") in the most recently reported study of Browman and Cartwright (1980), and running 92 kilometres in another study by Shapiro (1978 and 1979). It is not surprising that these two activities produce different effects on sleep patterns, although it is interesting that the changes induced by both these exercise events is in the same direction. If one considers sleep to be a recovery process for daily activity then the effect of exercise on sleep could be to the extent that it changes daily activity. Astrand (1976) has pointed out that "the 
basal metabolic rate in humans is quite high; 5,500 $7000 \mathrm{KJ}$ during a $24 \mathrm{~h}$ period being a realistic figure for an adult, which is equivalent to an extra energy demand of walking about 18 miles $(30 \mathrm{~km})^{\prime \prime}$. With this in mind it is not surprising that studies in which greater levels of exercise have been performed have produced (quantitatively) greater changes in sleep patterns. In other studies the changes after low levels of exercise have been confined to certain parts of the sleep recording, for example, the first sleep cycle (approximately the first one and a half hours of sleep). In these situations one may imagine that the recovery process has occurred in this time and therefore the remainder of sleep is unaltered.

On the basis of electro-encephalogram, electrooculogram, and electromyogram recordings, sleep can be divided into two major types: rapid-eye-movement (REM) sleep, which is associated with dreams, and nonREM (NREM) sleep. NREM can be subdivided into four stages. Stages 3 and 4 together are often referred to as slow-wave sleep (SWS), because of the high-amplitude, low-frequency waves seen on the EEG recording during these stages. As with most physiological parameters, the amounts of the components of sleep show a normal distribution, which is both age- and, to a lesser extent, sex-dependent. Other aspects of sleep that are commonly considered when studying sleep records include sleep latency (time from lights out to stage 2 sleep), sleep duration (total amount of sleep) and movement time (or percentage) during sleep. In 1970, Oswald suggested that slow-wave sleep (stages 3 and 4) is the most important component of the restorative function of sleep. Experiments have shown that the amount of slow-wave sleep is dependent on the duration of preceeding wakefulness (Webb and Agnew 1971). Human responsiveness (on the basis of a variety of measures) is lowest during SWS (for references see Oswald (1980)). If one considers metabolic activity, several factors that increase metabolism show increases of slow-wave sleep, and conversely decreased levels of SWS accompany decreased metabolism (Table 1).

On a basis of these observations and the prediction that SWS is the most significant component of the recovery process of sleep for daily activity, one would expect SWS to increase following exercise (provided that the amount of exercise is sufficient significantly to alter daily metabolism). This prediction implies a shift in the proportion of sleep occupied by its different components (i.e. an increase in 3 and 4 and a decrease of other stages). As mentioned above it is possible that this shift may be a temporal one and not only a quantitative one. There are other ways in which sleep may be more restorative. If total sleep time is longer then the absolute amount of SWS would be greater even with the proportion of the stages within sleep being constant. If this is the case one would expect

\section{TABLE I}

\section{Conditions ascociated with increased slow-wave sleep}

Youth - especially adolescence

Following exercise

Fit subjects

Prolonged prior wakefulness

Following sleep interruption

During and following chronic short sleep time

Short sleepers (proportionately)

Starvation

Anorexia nervosa on refeeding

Hyperthyroid patients

Hypothyroid patients on treatment

Following selective SWS depression

Following amino acid or glucose intake

Prolonged bed rest

Fenfluramine medication

\section{Conditions associated with decreased slow-wave sleep}

Advancing age

Quadriplegic patients

Blind subjects

Hypothyroid patients

Sheehan's syndrome

Selective depression of SWS (experimental)

longer sleep duration and possibly shorter sleep latency. A third alternative may be that there is some "intensity" component to SWS. An analogy can be made with REM sleep in which one may have two dream periods of equal duration but one of them may show a vastly increased number of actual eye movements in this given period, which may suggest an intensity component in the REM record. There are several metabolic correlates of SWS. Amongst the most notable of these is the synchrony of growth hormone release during SWS (Parker et al, 1969), and the low level of oxygen consumption during SWS (Brebbia and Altschuler, 1965). In animal studies the actual amplitude of the EEG has been shown to be greater (and frequency lower) in situations in which "intense" SWS may be expected (Borbely, 1978). Friedman, et al (1979) have also considered aspects of "sleep intensity" in rats, and did not find increased intensity with extra exercise after sleep deprivation. If there is an "intensity" component to SWS then it is quite possible that the proportion of SWS (i.e. SWS as a percentage of total night sleep) and absolute duration of SWS may remain constant and yet sleep may have a variable restorative value. Growth hormone (an anabolic and hence potentially "restorative" hormone) may be a component of this intensity factor of SWS. The observation by Adamson et al (1974), that growth hormone release during sleep is greater following exercise certainly gives credence to this notion, particularly as SWS was not increased in this study. In the only other study of this type, Zir et al (1971), showed no significant increase 
in SWS and no significant increase in growth hormone following light and moderate exercise. They did find that "four of ten subjects had greater post-exercise SWS" and "two of ten subjects had augmented post exercise sleep release of human growth hormone. This suggests that different subjects may respond in different ways. Some may increase the amounts or proportion of SWS, and some may increase intensity of SWS after exercise.

One important confounding variable affecting sleep after exercise is the stress induced by the exercise. If high levels of exercise are required to elicit the effect of exercise on SWS, then this exercise is likely to cause greater stress in unfit subjects. This may explain why the clearest demonstrations of increase in slow-wave sleep following exercise have been in fit subjects performing strenuous exercise. Furthermore, physical training may serve as a facilitatory factor for SWS expression, just as it facilitates a variety of physiological responses. There are other facets of training that may cause an increase in SWS; the increase in lean body mass has been suggested as one possibility by Trinder and Paxton (personal communication - 1980).

One may conclude that a variety of changes of sleep pattern may occur following exercise, which would be consistent with the model of sleep being restorative. These include: increase in sleep duration; decrease in sleep latency; decrease in movement during sleep; increase in SWS - possibly particularly in stage 4; and decrease in REM sleep and an associated delay till first appearance of REM. Stage 2 may increase as a component of increased duration of sleep and of a lower intensity restorative process; however, if there are very marked increases in SWS then stage 2 would probably be lower. There are no studies that show all of these changes, but almost all studies on the effect of exercise on sleep have shown these changes to some extent. The studies are very variable and some of the authors would probably not agree that their work support the hypothesis. However, when one considers that the EEG measure of SWS is really perhaps a crude approximation of the essence of the restorative nature of sleep, it would seem that the overall pattern of results that suggests greater recovery after exercise is quite definite.

The few studies that have addressed the question of subjective feeling after exercise have generally concluded that subjects feel more tired and more sleepy after exercise and, if the exercise is not too extreme, they feel that they sleep better. A different aspect of the interaction of sleep and exercise is the effect of the various sleep patterns on athletic performance. A diurnal variation in performance has previously been demonstrated by Rodahl et al (1976). Although no direct studies of this type have been carried out it would be reasonable to predict that, as with other physiological parameters that have a normal range, certain sleep patterns may be conducive to better athletic performance. This is supported by two types of studies. Firstly, in comparing fit and unfit subjects, Griffen and Trinder (1978) have shown greater levels of SWS in the fit subjects and Walker et al (1978) have shown that fitness is associated with reduced eye movement activity during sleep in normal subjects. These observations point to a sleep adaptation associated with fitness. Secondly, studies of sleep deprivation and sleep lack have shown changes in performance following disruption of sleep. The results of Donnell (1969) show that to detect performance impairment after sleep deprivation the shorter the deprivation the longer the performance test required. It has been shown by Davey (1973), that physical exertion and sleep deprivation interact to produce a deterioration of performance. Wilkinson, et al (1966) showed that "less than 5 hours sleep on a single night impaired vigilance; less than 3 hours impaired calculation".

There are many situations in which sleep is disturbed prior to an athletic event: recent change in time zone; excitement of the athlete causing early arousal; and early start of the event (especially an endurance (i.e. longer) event) requiring early arousal. All these factors will disturb sleep, and hence probably alter performance.

Some of the effects of time zone changes include repeated early morning awakenings (Evans et al, 1972) and particularly with eastward time zone changes there is more movement recorded during sleep and more Stage 1 sleep as well as less REM sleep (Hume, 1980). With westward changes there are more sleep stage shifts to wakening movement and Stage 1 and there is an increased time spent in REM sleep. The disruption of circadian rhythm which is pertinent to the athlete takes on average 5 or $\mathbf{6}$ days for re-entrainment to occur (Klein et al, 1970). This suggests that peak performance will not be achieved within approximately 1 week of a time zone change of 8 hours.

A final point relating to sleep in the athlete is that there are certain extreme endurance events that require marked sleep deprivation. It would seem prudent that these events have medical cover. In addition, an awareness by the athlete's "back-up team" of the potential effects of sleep deprivation on the athlete should be appreciated. This subject has been recently reviewed by Opstad et al (1978).

The effects of exercise on sleep can be summarised as follows. Exercise increases subjective tiredness, slowwave sleep, sleep duration and growth hormone release. It decreases sleep onset latency, and delays (and may decrease) REM sleep. 


\section{REFERENCES}

Adamson, L., Hunter, W. M., Ogunremi, R., Oswald, I. and Percy-Robb, I. W., 1974 "Growth hormone increase during sleep after daytime exercise". J.Endocrinol. 62: 473-478.

Åstrand, P., 1976 "Quantification of exercise capability and evaluation of physical capacity in man". Prog.Cardiovasc. Dis. 19: 51-67.

Borbely, A. A., 1978 Personal communication.

Brebbia, D. R. and Altschuler, K. Z., 1965 "Oxygen consumption rate and electroencephalographic stages of sleep". Science 150: 1621-1623.

Browman, C. P. and Cartwright, R. D., March 1980 "Exercise and sleep". Abstract APSS Sleep Conference, p. 144.

Davey, C. P., 1973 “Physical exertion and mental performance”. Ergonomics 16: 595-599.

Donnell, J. M., 1969 "Performance decrement as a function of total sleep loss and task duration". Percept.Mot.Skills 29: $711-714$.

Evans, J. I., Christie, G. A., Lewis, S. A., Daly, J. and Moore Robinson, S., 1972. Sleep and time-zone changes. Arch. Neurol. 26: 36-48.

Friedman, L., Bergmann, B. M. and Rechtschaffen, A., 1979 "Effect of sleep deprivation on sleepiness, sleep intensity and subsequent sleep in the rat". Sleep 1: 369-391.

Griffen, S. J. and Trinder, J., 1978 "Physical fitness, exercise and human sleep". Psychophysiology 5: 447-450.

Hume, K. I., 1980. Sleep adaptation alter phase shifts of the sleep-wakefulness rhythm in man. Sleep 2: 417-435.

Klein, K. E., Brumer, H. and Haltmann, H., 1970. Circadian rhythms of pilot's efficiency and effects of multiple time-zone travel. Aerospace Med. 41: 125-232.

Opstad, P. K. E., Kanger, R., Nummestad, M. and Raabe, W., 1978 "Performance, mood and clinical symptoms in men exposed to prolonged severe physical work and sleep deprivation". Aviat.Space.Environ.Med. 49: 1065-1073.

Oswald, I., 1970 "Sleep the great restorer". New Scientist 46: 170-172.

Oswald, I. and Corner, M., 1980 "Sleep as a restorative process: human clues". Progress in Brain Research, Elsevier, Amsterdam.

Parker, P. K., Sassin, J. F., Mace, J. W., Gotlin, R. W. and Rossmann, L. G., 1969 "Human growth hormone release during sleep-electroencephalographic correlation". J.Clin.Endocrị. 29: 871-874.

Rodahl, A., O'Brien, M. and Firth, R. G. R., 1976 "Diurnal variation in performance of competitive swimmers". J.Sports Med.\& Phys. Fitness 16: 72-76.

Shapiro, C. M., 1978 "Sleepiness of the long-distance runner". J.Physiol.(Lond). 276:50-51.

Shapiro, C. M., 1979 "Effect of exercise on sleep". S.A. Sports Medicine 6: 9-12.

Walker, J. M., Floyd, T. C., Fein, G., Cavness, C., Lualhatti, R. and Feinberg, I., 1978 "Effects of exercise on sleep". J.Appl.Physiol. $44:$ 945-951.

Webb, W. B. and Agnew, M. W., 1971 "Stage four sleep: influence of time course variables". Science 174: $1354-1356$.

Wilkinson, R. T., Edwards, R. S. and Haines, E., 1966 "Performance following a night of reduced sleep". Psychological Science 5: 471-472. 
Zir, L. M., Smith, R. A. and Parker, D. C., 1971 "Human growth hormone release in sleep: effect of daytime exercise J.Clin.Endocrinol.Metab. 32: 622-665.

\section{DISCUSSION}

Dr. Bentley: I'd like to ask Dr. Shapiro about the use of sleeping tablets by people travelling away to events. I'm often asked for tablets by such people, and l'd like to know if he has any views on the various kinds.

Dr. Shapiro: Sleeping tablets do alter sleep patterns, producing particularly a reduction in REM sleep. In general, I'd say it is undesirable to take sleeping tablets, particularly very close to the event; the effects carry over for several day? depending on how long the tablets have been taken for. The levels of some of the drugs build up in the system and, for example, skilled activity may be impaired for quite a time after the tablets are taken. A real problem may exist i before an event, the subject is excited and unable to sleep without sleeping tablets; this may happen for instance, in university student before an examination. I wouldn't like to give a definite recommendation on this. Ideally, what one is looking for is a short-acting drug that will allow people to fall asleep quickly but have no further effect, and I'm surg this will be achieved in the future. 